\section{Erhöhen Symptome der Hüfte bei Arthrose die Sterblichkeit?}

Cleveland RJ et al. Hip symptoms are associated with premature mortality: the Johnston County Osteoarthritis Project. Osteoarthritis Cartilage 2020; 28: 1330-1340

Osteoarthrose tritt häufig im Hüftgelenk auf. Schmerzen und Bewegungseinschränkungen sind die Folge. Dies kann zu Einschränkungen im Alltag und der Mobilität führen. Meist treten begleitende Komorbiditäten auf, welche die Sterblichkeit erhöhen. Die Autoren möchten den Zusammenhang zwischen Hüft-Arthrose und der möglichen erhöhten Sterblichkeit untersuchen. Dazu soll eine Kohortenstudie mit bis zu 25 Jahren Nachverfolgungsdauer ausgewertet werden.

Die Daten stammen aus dem Johnston County Osteoarthritis Projekt, einer Community-basierten prospektiven Kohortenstudie zu Osteoarthrose in North Carolina. Die Kohorte $(n=3919)$ setzt sich aus Teilnehmern in sechs Gemeinden zusammen, welche hauptsächlich Afroamerikaner oder Kaukasier sind. Die Probanden müssen bei Studieneintritt mindestens 45 Jahre alt sein. Die erste Welle wurde von 1991 bis 1998 rekrutiert, die zweite Welle von 2003 bis 2004 um Ausfälle auszugleichen. Alle Frauen ab 50 Jahren und alle Männer wurden einer Hüftradiografie zu Beginn und bei jedem Nachverfolgungszeitpunkt unterzogen. Die Hüftarthrose wird nach dem Kellgren-Lawrence-Score eingeteilt. Arthrose wurde ab einem Wert von 2 oder höher definiert. Das Auftreten von Hüftsymptomen wurde abgefragt. In die statistische Auswertung wurden diverse Kovariaten aufgenommen, z. B. Alter, Geschlecht, Rasse, Bildung, Drogenkonsum, weitere Erkrankungen und Werte weiterer Fragebögen. Die Nachverfolgung fand zwischen 1999 und 2004 (T1), zwischen 2006 und 2010 (T2) und zwischen 2013 und 2015 (T3) statt. Verstorbene wurden anhand von ICD kodiert. Die statistische Auswertung inklusive Sensitivitätsanalysen ist detailliert in der Studie beschrieben.
Das Durchschnittsalter lag bei 62,3 Jahren, $61 \%$ waren weiblich und $33 \%$ afroamerikanischer Herkunft. Zu Beginn hatten $45 \%$ der Probanden weder Hüftarthrose noch Symptome im Hüftgelenk, 19\% wiesen nur eine Hüftarthrose auf. Weiterhin zeigten sich bei $25 \%$ nur Symptome der Hüfte, während $11 \%$ sowohl eine Arthrose als auch Symptome hatten. Die mittlere Nachverfolgungsdauer lag bei 14,2 Jahren. Insgesamt starben 1762 Probanden, 661 davon an Herzkreislauferkrankungen. Die Auswertung der auf Alter und Geschlecht adjustierten Kaplan-Meier Überlebenskurven, zeigt ähnliche Ergebnisse für Probanden mit Arthrose und Schmerzen sowie Probanden nur mit Symptomen. Ähnliche Ergebnisse gibt es auch für Teilnehmer nur mit Arthrose sowie Teilnehmer ohne Symptome und ohne Arthrose. Die Cox Regression ergaben ein erhöhtes Todesrisiko für Teilnehmer nur mit Symptomen im Hüftgelenk. Die Autoren schlussfolgern, dass die erhöhte Sterblichkeit mit größeren Einschränkungen und Komorbiditäten in Zusammenhang steht.

\section{FAZIT}

Das Hüftgelenk ist bei Osteoarthrose oft betroffen. Schmerzen und Bewegungseinschränkung belasten den Alltag und können zu Immobilität und weiteren Einschränkungen führen, welche auch mit Komorbiditäten einhergehen. Die Autoren untersuchten in einer prospektiven Kohortenstudie den Zusammenhang zwischen Arthrose und Sterblichkeit. Sie konnten eine erhöhte Sterblichkeit für Menschen mit Hüftsymptomen aber ohne Arthrose im Hüftgelenk feststellen. Dies sollte zur Prävention genutzt werden.

Stephanie Burgess, Stuttgart 